\title{
Criptococose mamária manifesta após corticoterapia
}

\author{
Cryptococcal mastitis after corticosteroid therapy
}

\author{
Sória Ramos-Barbosa ${ }^{1}$, Luciana Silva Guazzelli $\quad{ }^{1}$ e Luiz Carlos Severo ${ }^{1}$
}

\section{RESUMO}

Descrevemos caso de criptococose mamária em paciente de 46 anos em uso de corticoterapia. A micose foi diagnosticada por bistopatologia e detecção de antígenos criptocócicos. Destacamos o dilema diagnóstico do granuloma sarcóide e a rara manifestação de mastite criptocócica.

Palavras-chaves: Criptococose. Sarcoidose. Mastite criptocócica. Granuloma.

\section{ABSTRACT}

We described a case of cryptococcal mastitis in a 46 years old female patient receiving corticosteroids for sarcoidosis. There was radiological pulmonary deterioration and a cystic lesion was found in the left breast. The mycosis was diagnosed by histopathology and cryptococcal antigens. We emphasized the sarcoid granuloma diagnostic's dilemma an the rare manifestation of cryptococcal infection as mastitis.

Key-words: Cryptococcosis. Sarcoidosis. Cryptococcal mastitis. Granuloma.

A criptococose é adquirida por inalação e é conhecido o marcado tropismo do fungo pelo sistema nervoso central, sendo a principal manifestação clínica desse acometimento a meningoencefalite. Contudo, qualquer órgão do corpo pode ser acometido. 0 principal fator predisponente é o defeito na imunidade celular, tais como o verificado na Aids e na imunossupressão terapêutica ${ }^{5}$.

Apresentaremos caso de criptococose mamária secundária a corticoterapia, em paciente com diagnóstico operacional de sarcoidose.

\section{RELATO DO CASO}

Paciente feminina, branca, 46 anos, procedente de Novo Hamburgo (RS), notou há dois meses tumefação na fossa ilíaca esquerda, associada 'a dor em região lombar irradiada para abdome, além de dispnéia. Referia anorexia, emagrecimento de 10kg em 4 meses, cefaléia matinal com localização occipital e parietal. Negava febre ou sintomas cardiovasculares. 0 exame físico revelou linfadenomegalia difusa e esplenomegalia. A telerradiografia do tórax mostrou infiltrado intersticial micronodular em ambos os pulmões, predominante a direita e nas metades superiores. A sorologia para vírus HIV foi negativa e a glicemia de jejum foi de $95 \mathrm{mg} / \mathrm{dl}$. A biópsia de gânglio cervical em cortes corados à hematoxilina-eosina evidenciou a presença de inflamação granulomatosa de tipo tuberculóide, sem necrose caseosa.

0 tipo das lesões pulmonares sugeriu processo inflamatório granulomatoso crônico. 0 aspecto histopatológico do gânglio cervical e a anergia à tuberculina levaram à hipótese operacional de sarcoidose, sendo introduzido o uso de corticóide: prednisona $30 \mathrm{mg} / \mathrm{dia} / 1$ semana, seguido de $20 \mathrm{mg} / \mathrm{dia}$, sem melhora da linfadenomegalia. Após 4 meses, foi reduzido o corticóide para $10 \mathrm{mg} /$ dia e notou-se, ao exame físico, nódulo doloroso em mama esquerda. Após mamografia, foi realizada exérese do nódulo mamário. 0 estudo anatomopatológico mostrou lesão cística contendo grande número de elementos fúngicos, esféricos encapsulados característicos de Cryptococcus neoformans. Nova telerradiografia do tórax nesta ocasião mostrou cavitações múltiplas parenquimatosas em ambos os pulmões. 0 exame sorológico evidenciou antígenos criptocócicos em título de 1:512.

1. Universidade Federal do Rio Grande do Sul, Porto Alegre, RS.

Endereço para correspondência : Dr. Luiz Carlos Severo. Laboratório de Micologia/Hospital Santa Rita, Santa Casa - Complexo Hospitalar Annes Dias 285, $90020-090$ Porto Alegre, RS.

Fax: $55513214-8435$

email:severo@santacasa.tche.br

Recebido para publicação em 30/12/2002

Aceito em 6/12/2003 
Houve boa resposta ao tratamento antifúngico (cetoconazol $100 \mathrm{mg}, 12 / 12 \mathrm{~h}$ por um ano) com regressão das lesões pulmonares e da linfadenomegalia.

\section{DISCUSSÃO}

A demonstração de granuloma não caseoso, a linfadenopatia, 0 envolvimento pulmonar bilateral levantaram a hipótese diagnóstica de sarcoidose. Contudo, a falta de resposta clínica à corticoterapia e o surgimento de lesão tumefasciente mamária fizeram com que a criptococose fosse diagnosticada. Este fato, associado ao sucesso terapêutico com antifúngico colocaram em dúvida o diagnóstico de sarcoidose.

A sarcoidose, devido a inversão da relação CD4/CD8, consequência da redistribuição dos linfócitos T auxiliares do sangue periférico para os pulmões, determina imunodepressão relativa, o que facilita a infecção por germes oportunistas de célula T, como é o caso do C. neoformans ${ }^{2}$, principalmente após corticoterapia ${ }^{79}$.

A criptococose mamária foi inicialmente descrita em animais ${ }^{6}$; em humanos restringe-se a uma dezena de relatos ${ }^{4}$, em sua maioria com lesões de disseminação, especialmente para sistema nervoso central. A mama, por tratar-se de glândula apócrina, favorece 0 crescimento fúngico ${ }^{3}$. A manifestação da lesão mamária pode ser a apresentação inicial da micose ou de doença sistêmica manifesta ${ }^{4}$.

Por sua vez, a sarcoidose é grande mimetizadora de infecções respiratórias ${ }^{1}$, especialmcnte fúngicas, sendo que o granuloma não caseoso, observado nesta doença, e seus achados laboratoriais podem ser encontrados também em micoses sistêmicas ${ }^{1011}$, particularmente na histoplasmose onde há elevação dos níveis da enzima conversora de angiotensina, que é utilizada para avaliar a evolução da sarcoidose ${ }^{1112}$.
Por fim, a evolução clínica e radiológica e a refratariedade ao tratamento com corticóides em paciente com sarcoidose estável deve suscitar a possibilidade de infecção superimposta ${ }^{18}$, ou infecção fúngica não diagnosticada, ou mesmo doença fúngica produzindo resposta inflamatória crônica similar à sarcoidose ${ }^{11}$.

\section{REFERÊNCIAS BIBLIOGRÁFICAS}

1. Blackmon GM, Raghu G. Pulmonary sarcoidosis: a mimic of respiratory infection. Seminars in Respiratory Infections 10:176-186, 1995.

2. Botha RJP, Wessels E. Cryptococcal meningitis in an HIV negative patient with systemic sarcoidosis. Journal of Clinical Pathology 52:928-930, 1999.

3. Casadevall A, Perfect JR. Cryptococcus neoformans. ASM Press, Washington. p. 407-456, 1998.

4. Golmann M, Pottage JC.Cryptococcal infection of the breast. Clinical Infectious Diseases 21:1166-1169, 1995.

5. Kwon-Chung KJ, Bennett JE. Cryptococcosis. In: Kwon-Chung KJ, Bennett JE (eds) Medical mycology. $1^{\text {st }}$ edition, Lea \& Febiger, Philadelphia, p. 397-446, 1992.

6. Littman ML, Zimmerman LE. Cryptococcosis. Torulosis or european blastomycosis. Grune \& Stratton. New York, p. 38-46, 1956.

7. Nottebart HC, McGehee RF, Utz JP. Cryptococcosis complicating sarcoidosis. American Review of Respiratory Disease 107:1060-1063, 1973.

8. Shijubo N, Fujishima T, Ooashi K, Morita S, Shigehara K, Nakata H, Abe S Pulmonary cryptococcal infection in an untreated patient with sarcoidosis. Sarcoidosis 12:71-74, 1995

9. Sokolowski JW, Shillaci RF, Motley TE. Disseminated crytococcosis complicating sarcoidosis. American Review of Respiratory Disease 100: 717-722, 1969.

10. Wanke B, Londero AT. Paracoccidioides brasiliensis. In: Ajello L, Hay RJ (eds) Medical mycology, Arnold, London, vol 4, p. 395-407, 1988.

11. Wheat LJ, French LV, Wass JL. Sarcoidlike manifestations of histoplasmosis. Archives of Internal Medicine 149:2421-2426, 1989.

12. Yassen Z, Havlichek D, Mathes B, Hahn M-H. Disseminated histoplasmosis in a patient with sarcoidosis; a controversial relationship and a diagnostic dilemma. The American Journal of the Medical Sciences 313:187-190, 1997. 